\title{
Intersubband Relaxation in Step Quantum Well Structures
}

\author{
J. P. SUN ${ }^{\mathrm{a}, *}$, H. B. TENG ${ }^{\mathrm{a}}$, G. I. HADDAD ${ }^{\mathrm{a}}$, M. A. STROSCIO ${ }^{\mathrm{b}}$ and G. J. IAFRATE ${ }^{\mathrm{b}}$ \\ ${ }^{\text {a }}$ Solid State Electronics Laboratory, Department of Electrical Engineering and Computer Science, \\ The University of Michigan, Ann Arbor, Michigan, 48109-2122; \\ ${ }^{\mathrm{b}}$ U.S. Army Research Office, P.O. Box 12211, Research Triangle Park, \\ North Carolina, 27709-2211
}

\begin{abstract}
Intersubband relaxation due to electron interactions with the localized phonon modes plays an important role for population inversion in quantum well laser structures designed for intersubband lasers operating at mid-infrared to submillimeter wavelengths. In this work, intersubband relaxation rates between subbands in step quantum well structures are evaluated numerically using Fermi's golden rule, in which the localized phonon modes including the asymmetric interface modes, symmetric interface modes, and confined phonon modes and the electron-phonon interaction Hamiltonians are derived based on the macroscopic dielectric continuum model, whereas the electron wave functions are obtained by solving the Schrödinger equation for the heterostructures under investigation. The sum rule for the relationship between the form factors of the various localized phonon modes and the bulk phonon modes is examined and verified for these structures. The intersubband relaxation rates due to electron scattering by the asymmetric interface phonons, symmetric interface phonons, and confined phonons are calculated and compared with the relaxation rates calculated using the bulk phonon modes and the Fröhlich interaction Hamiltonian for step quantum well structures with subband separations of $36 \mathrm{meV}$ and $50 \mathrm{meV}$, corresponding to the bulk longitudinal optical phonon energy and interface phonon energy, respectively. Our results show that for preferential electron relaxation in intersubband laser structures, the effects of the localized phonon modes, especially the interface phonon modes, must be included for optimal design of these structures.
\end{abstract}

Keywords: Localized phonons, relaxation rates, intersubband laser

\section{INTRODUCTION}

In recent years, novel tunneling injection lasers using structures based on semiconductor quantum wells have provided the means for achieving lasing wavelengths from the mid-infrared to the submillimeter wave region $[1,2,3]$. These lasers incorporate narrow quantum well regions that

* Corresponding author. 
must have thicknesses as small as $30-50 \AA$ and have transition level separations engineered to one unit or a multiple of bulk Longitudinal-Optical (LO) phonon energy. It is now well known that the shape and energies of LO phonon modes are modified by quantum wells. Specifically, LO phonons in quantum wells may be described by the dielectric continuum model in terms of confined and interface modes that have properties different from those of bulk phonons $[4,5,6]$. In this paper, the interface and confined LO phonon modes of the continuum model [7] are used with Fermi's golden rule to calculate relaxation rates between quasi bound states in step quantum well structures. We show that the electron relaxation rates are dominated by the interface phonon scattering, and that the symmetries of phonon modes induce inherent selection rules of transitions among well levels. In addition, the sum rule for the form factors [6] is verified numerically for the step quantum well structures.

\section{INTERFACE PHONON ASSISTED TRANSITION}

The well structure in this study is shown in Figure 1 , denoting the innermost $\mathrm{GaAs}$ to be layer 1, the next $\mathrm{Al}_{0.25} \mathrm{Ga}_{0.75} \mathrm{As}$ layer 2 and the outermost $\mathrm{Al}_{0.6} \mathrm{Ga}_{0.4} \mathrm{As}$ layer 3 . There are six types of phonon modes due to the double interfaces between layers 1 and 2: (1) symmetric interface modes, (2) antisymmetric interface modes, (3) confined LO modes, (4) confined TO modes, and (5) half-space LO modes. With the eigenmodes given by [6], we can obtain the electron-phonon interaction from the Fröhlich continuum model

$$
H_{e-p}=e \int d^{2} r \int d z \rho(\mathbf{r}, z) \phi(\mathbf{r}, z)
$$

where $\rho(\mathbf{r}, z)$ is the generalized electron density operator and $\phi(\mathbf{r}, z)$ the electrostatic potential created by the LO phonons. Since wavefunctions of the first two levels have opposite parities,

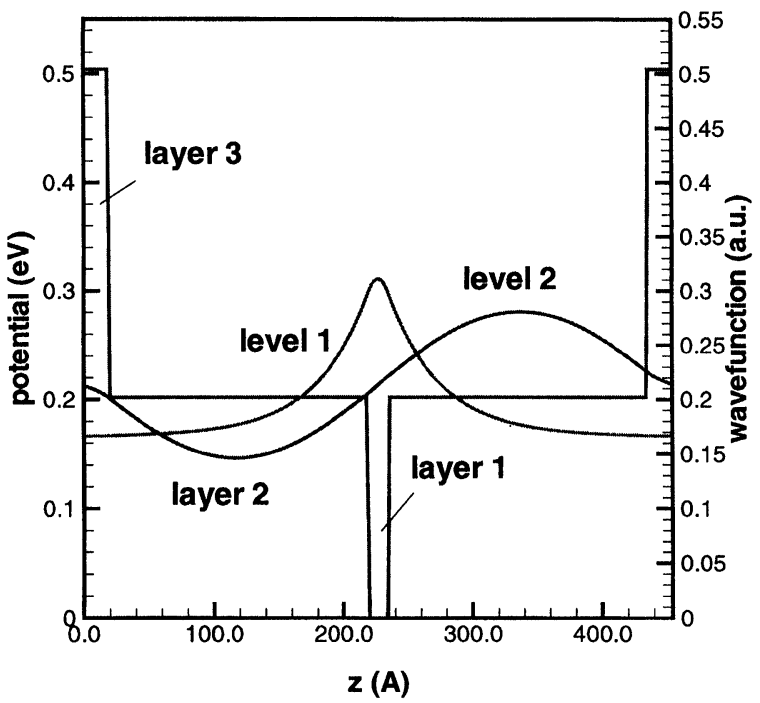

FIGURE 1 Well potential and wavefunctions of the first two levels.

only the antisymmetric modes may contribute significantly to electron transition rates between these two levels. For the antisymmetric phonon modes,

$$
\begin{aligned}
H_{A_{-}^{+}}= & \Sigma_{q}\left[\frac{\hbar \omega_{A_{-}^{+}} e^{2}}{2 \varepsilon_{0} L^{2}}\right]^{\frac{1}{2}}\left[\beta_{1}^{-1}\left(\omega_{A_{-}^{+}}\right) \operatorname{coth}\left(\frac{1}{2} q a\right)\right. \\
& \left.+\beta_{2}^{-1}\left(\omega_{A_{-}^{ \pm}}\right)\right]^{-\frac{1}{2}} \\
& \times \frac{1}{\sqrt{2 q}} e^{i q r} f_{A}(q, z)\left[a_{A_{-}^{ \pm}}(q)+\text { h.c. }\right],
\end{aligned}
$$

where $q$ is the two dimensional phonon wavevector parallel to the heterostructure interface,

$$
\beta_{n}(\omega)=\left[\frac{1}{k_{\infty n}}-\frac{1}{k_{0 n}}\right] \frac{\omega_{L n}^{2}}{\omega^{2}}\left[\frac{\omega^{2}-\omega_{T n}^{2}}{\omega_{L n}^{2}-\omega_{T n}^{2}}\right]^{2}, \quad n=1,2
$$

where $\omega_{T n}$ and $\omega_{L n}$ are the frequencies of the longitudinal optical (LO) phonon and the transverse-optical (TO) phonon, respectively. $\omega_{A_{-}^{+}}$are the antisymmetric mode frequencies and are given 
by solutions of the following equation,

$$
\varepsilon_{1}(\omega) \operatorname{coth}\left(\frac{1}{2} Q a\right)+\varepsilon_{2}(\omega)=0
$$

For long wavelengths they approach $\omega_{L 1}$ and $\omega_{T 2}$. $f_{A}$ is defined as

$$
f_{A}(q z)= \begin{cases}-e^{q\left(z+\frac{1}{2} a\right)}, & z \leq-\frac{1}{2} a \\ \sinh (q z) / \sinh \left(\frac{1}{2} q a\right), & -\frac{1}{2} a \leq z \leq \frac{1}{2} a \\ e^{q\left(z-\frac{1}{2} a\right)} . & z \geq \frac{1}{2} a\end{cases}
$$

The electron-phonon interaction induces electron transition between the two well levels. Using Fermi's golden rule, the rates of transitions assisted by various modes of the interface phonon and bulk phonon are calculated as functions of electron in-plane energy. In Figure 2, numerical results of the antisymmetric and bulk phonon assisted transitions are given at a well level energy separation of $E_{2}-E_{1}=47 \mathrm{meV}$, which shows that the antisymmetric interface phonon assisted transition dominates at a level separation close to the antisymmetric phonon frequency. Contributions from the other phonon modes are generally negligible.

The maximum transition rates occur when the electron in-plane energy compensates the difference between the mode frequency and energy level separation. Figure 3 presents a comparison of the maximum transition rates of the bulk mode and antisymmetric mode at various values of $E_{2}-E_{1}$, which are obtained by varying the quantum well width. A device structure which is engineered with an energy level separation of the antisymmetric interface mode frequency would have a relaxation rate several times larger than that with a bulk mode frequency separation, as shown in Figure 3.

For the structure under study, the upper antisymmetric mode plays the most important role in the transition between the energy level 2 and level 1. For other transitions (e.g. between level 3 and level 1) the symmetric modes may become important. The selection rule is due to well



FIGURE 2 Transition rates of antisymmetric and bulk modes.

defined parities of the antisymmetric and symmetric modes. In most cases, it is also applicable to non-symmetric well structures to a certain degree, but the specific phonon modes differ in different device structures and will have to be derived for a specific structure.

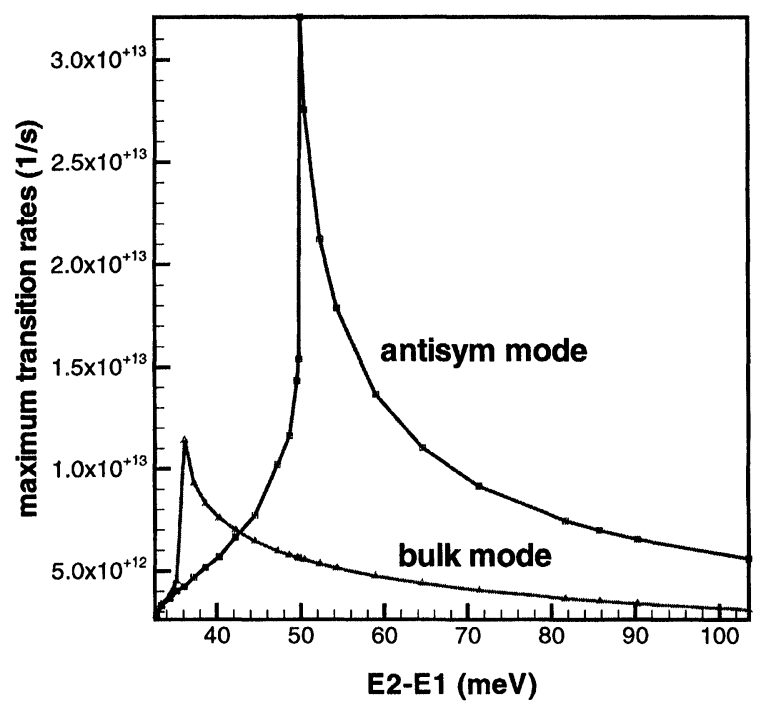

FIGURE 3 Resonant transition rates. 


\section{SUM RULE OF FORM FACTORS}

The form factors [6] can be defined through effective electron-electron interaction due to exchange of optical phonons. The form factors are independent of material parameters but depend on the spatial shape of the phonon modes and subband electron wavefunctions. In this double heterostructure, the form factors for a subband and different phonon modes are given by

$$
\begin{aligned}
& F_{S}(q)=\frac{1}{1+\tanh \left(\frac{1}{2} q a\right)}\left[\int_{-\infty}^{\infty} d z|\psi(z)|^{2} f_{S}(q z)\right]^{2}, \\
& F_{A}(q)=\frac{1}{1+\operatorname{coth}\left(\frac{1}{2} q a\right)}\left[\int_{-\infty}^{\infty} d z|\psi(z)|^{2} f_{A}(q z)\right]^{2}, \\
& F_{C}(q)=\int_{-\frac{a}{2}}^{\frac{a}{2}} d z \int_{-\frac{a}{2}}^{\frac{a}{2}} d z^{\prime}|\psi(z)|^{2}\left|\psi\left(z^{\prime}\right)\right|^{2} \\
& {\left[e^{-q\left|z-z^{\prime}\right|}-\frac{1}{1+\tanh \left(\frac{1}{2} q a\right)} \frac{\cosh (q z) \cosh \left(q z^{\prime}\right)}{\cosh ^{2}\left(\frac{1}{2} q a\right)}\right.} \\
& \left.-\frac{1}{1+\operatorname{coth}\left(\frac{1}{2} q a\right)} \frac{\sinh (q z) \sinh \left(q z^{\prime}\right)}{\sinh ^{2}\left(\frac{1}{2} q a\right)}\right] \text {, } \\
& F_{H+}(q)=\int_{\frac{a}{2}}^{\infty} d z \int_{\frac{a}{2}}^{\infty} d z^{\prime}|\psi(z)|^{2}\left|\psi\left(z^{\prime}\right)\right|^{2} \\
& {\left[e^{-q\left|z-z^{\prime}\right|}-\left[e^{-q\left|z+z^{\prime}-a\right|}\right],\right.} \\
& F_{H-}(q)=\int_{-\infty}^{-\frac{a}{2}} d z \int_{-\infty}^{-\frac{a}{2}} d z^{\prime}|\psi(z)|^{2}\left|\psi\left(z^{\prime}\right)\right|^{2} \\
& {\left[e^{-q\left|z-z^{\prime}\right|}-\left[e^{-q\left|z+z^{\prime}+a\right|}\right]\right. \text {. }}
\end{aligned}
$$

where $f_{\mathrm{S}}$ is given by

$$
f_{S}(q z)= \begin{cases}e^{q\left(z+\frac{1}{2} a\right)}, & z \leq-\frac{1}{2} a \\ \cosh (q z) / \cosh \left(\frac{1}{2} q a\right), & -\frac{1}{2} a \leq z \leq \frac{1}{2} a \\ e^{q\left(z-\frac{1}{2} a\right)} . & z \geq \frac{1}{2} a\end{cases}
$$

These form factors always satisfy the sum rule [6]

$$
\begin{aligned}
F_{B}(q) & =F_{S}(q)+F_{A}(q)+F_{C}(q) \\
& +F_{H+}(q)+F_{H-}(q)
\end{aligned}
$$

which is a direct consequence of the complete orthonormality of the interface eigenmodes. Ver- ifying the sum rule provides a justification of our numerical calculations. The sum rule between the various form factors has been verified for different well levels of the step quantum well structure. As an example, in Figure 4, the calculated form factors for level 2 are plotted. The sum of form factors of the symmetric mode and half space modes is indeed equal to that of the bulk mode. The form factors for the confined LO mode and antisymmetric mode in this case are negligible compared to those for the symmetric and half space $(H+$ and $H-)$ modes, although this is not always true for other levels.

\section{CONCLUSION}

By examining and comparing electron transition rates induced by bulk and interface phonon modes, our calculations for step quantum well structures designed for intersubband lasers have shown that, to facilitate preferential electron relaxation, it is important to consider the transition induced by the interface phonon modes and have electron subbands engineered with their separation close to the interface phonon energy in the device structures.

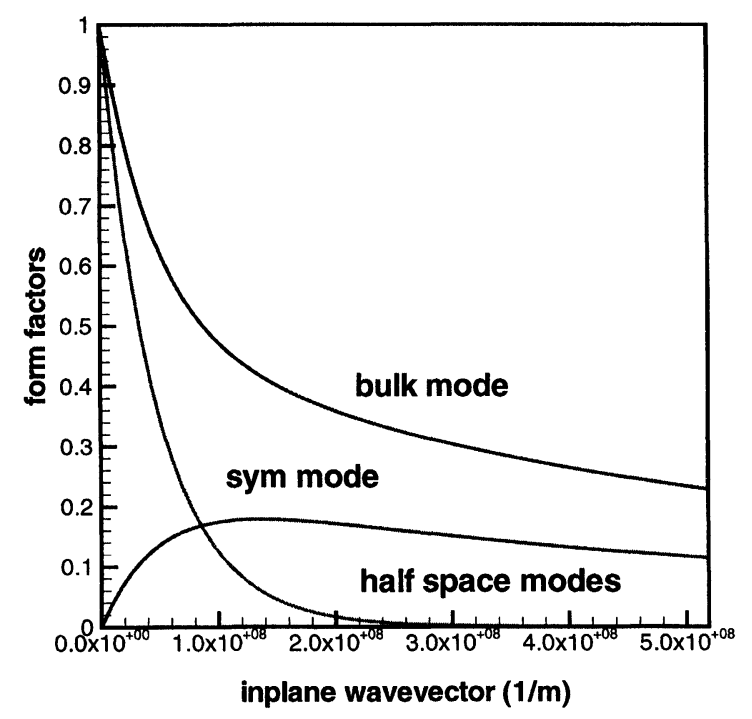

FIGURE 4 Sum rule of form factors. 


\section{References}

[1] Sun, H. C., Davis, L., Sethi, S., Singh, J. and Bhattacharya, P. (1993). IEEE Photonics Tech. Lett., 5, 870.

[2] Bhattacharya, P., Singh, J., Yoon, H., Gutierrez-Aitken, A. L., Jambunathan, R., Davis, L. and Sun, H. C. (1994). IEEE LEOS Newsletter, 20, August 1995; Davis, L., Sun, H. D., Yoon, H. and Bhattacharya, P. Appl. Phys. Lett., 64, 3222.

[3] Faist, J., Capasso, F., Sivco, D. L., Sirtori, C., Hutchinson, A. L. and Cho, A. Y. (1994). Science, 264, 553.

[4] Fuchs, R. and Kliewer, K. L. (1966). Phys. Rev., 140, A2076 (1965); Kliewer, K. L. and Fuchs, R. Phys. Rev., 144, 495.

[5] Licari, J. J. and Evrard, R. (1977). Phys. Rev., B15, 22345.

[6] Mori, N. and Ando, T. (1989). Phys. Rev., B40, 6175.

[7] Stroscio, M. A. (1996). J. Appl. Phys., 80, 6864.

\section{Authors' Biographies}

J.P. Sun received his B.S. degree from Nanjing Institute of Technology, China, and M.S. and Ph.D. degrees from the University of Michigan, all in Electrical Engineering. He has worked at Nanjing Solid State Devices Institute, China, Electronics Research Laboratory, University of California at Berkeley, and Solid State Electronics Laboratory, The University of Michigan, where he is now an Assistant Research Scientist. His current research interests include modeling of electronic and photonic devices based on quantum well structures.

H. B. Teng received his B.S. degree from University of Science and Technology of China and M.S. degree from Institute of Theoretical Physics, Chinese Academy of Science, both in physics, and his M.S. degree from the University of Michigan in Electrical Engineering. $\mathrm{He}$ is currently a $\mathrm{Ph} . \mathrm{D}$. student of The University of Michigan working on electronic and photonic device modeling.

G. I. Haddad received the B.S.E., M.S.E. and $\mathrm{Ph} . \mathrm{D}$. degrees in electrical engineering from the University of Michigan. He is currently the Robert J. Hiller Professor and Chairman of the Electrical
Engineering and Computer Science Department and Director of the Center for High Frequency Microelectronics, The University of Michigan. His current research areas are microwave and millimeter-wave solid-state devices and monolithic integrated circuits, microwave-optical interactions and optoelectronic devices and integrated circuits. Among numerous awards he received, he is the recipient of the 1996 IEEE-MTT Distinguished Educator Award. He is a member of the American Society for Engineering Education, and the American Physical Society. He is a Fellow of the IEEE and a member of the National Academy of Engineering.

Dr. Michael A. Stroscio has published widely in the field of physics and in recent years has specialized in quantum transport and phonon effects in dimensionally-confined structures. He has published about 140 journal articles. He is the Principal Scientist at he U.S. Army Research Office. Dr. Stroscio is a Fellow of the IEEE and a Fellow of the Yale Science and Engineering Association. $\mathrm{He}$ is an Adjunct Professor at Duke University and at the North Carolina State University.

Dr. Gerald J. Iafrate has published widely in the field of solid-state electronics and has made seminal contributions in quantum transport theory. He has published about 140 journal articles in these fields. He is the former Director of the U.S. Army Research Office and the first U.S. Army Senior Scientist of modern times; he was also the Principal Research Scientist at the U.S. Army electronics Technology and Device Laboratory. Dr. Iafrate is a Fellow of the IEEE, a Fellow of the APS, and a Fellow of the AAAS. Dr. Iafrate is a Fellow of the Polytechnic University and he is now Professor of Electrical Engineering Department at Notre Dame University. 

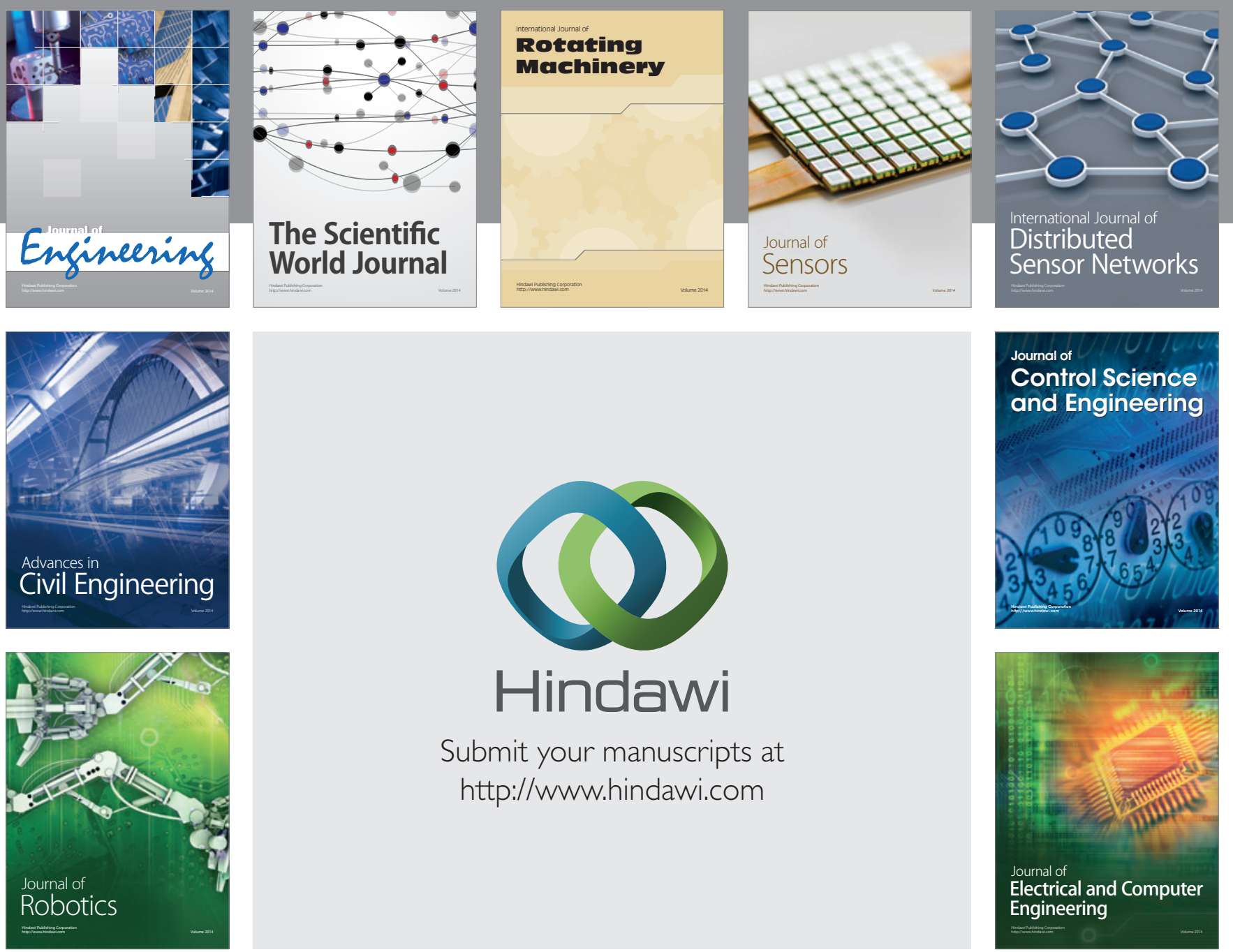

Submit your manuscripts at

http://www.hindawi.com
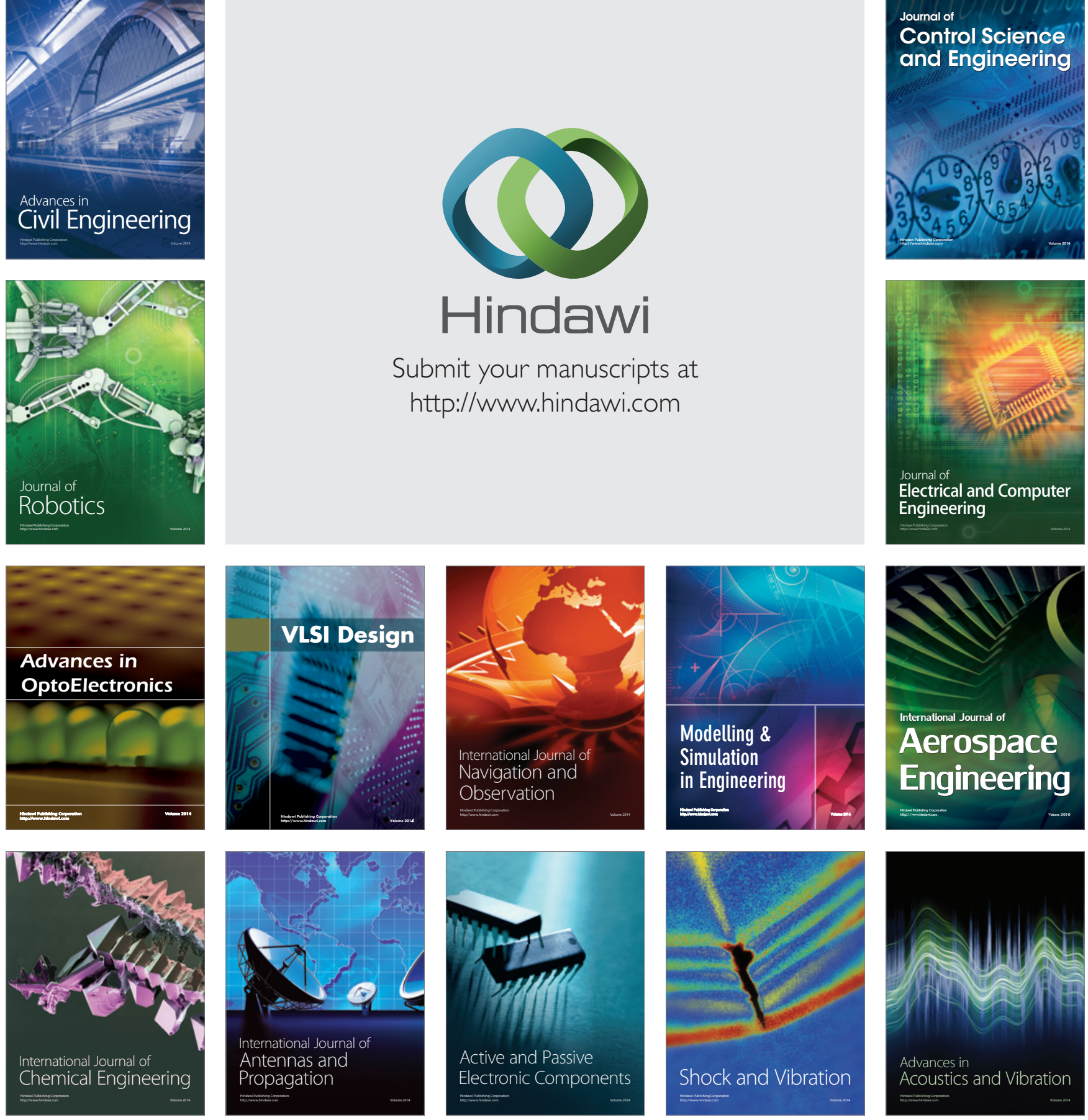